\title{
Symmetry breaking in Leidenfrost flows
}

Ambre Bouillant, Timothée Mouterde, Philippe Bourrianne, Christophe Clanet, and David Quéré Physique \& Mécanique des Milieux Hétérogènes, UMR 7636 du CNRS, ESPCI,

PSL Research University, 75005 Paris, France

and LadHyX, UMR 7646 du CNRS, École Polytechnique, 91128 Palaiseau, France

(Received 26 August 2018; published 17 October 2018)

This paper is associated with a video winner of a 2017 APS/DFD Milton van Dyke Award for work presented at the DFD Gallery of Fluid Motion. The original video is available from the Gallery of Fluid Motion, https://doi.org/10.1103/ APS.DFD.2017.GFM.V0052

DOI: 10.1103/PhysRevFluids.3.100502

Drops of volatile liquid placed on sufficiently hot plates are spectacularly mobile and move away from their initial position, as reported in 1756 by Leidenfrost [1]. The elusive character of Leidenfrost drops arises from the presence of a cushion of vapor beneath the liquid, which generates a frictionless situation and makes these hovercrafts sensitive to any external force (gravity, airflows). This "passive" behavior is questioned here. Indeed, even if the Leidenfrost phenomenon has been described for about a quarter of a millennium, the existence and the effect of internal flows were hardly addressed. To investigate such motions, we look at the drops while they are pinned by a needle or immobilized by deepening the substrate. Figure 1 shows the presence of strong internal flows, as revealed by long-time exposure (about $200 \mathrm{~ms}$ ) photos of water drops containing tracers placed on brass brought to $300{ }^{\circ} \mathrm{C}$.

(a)

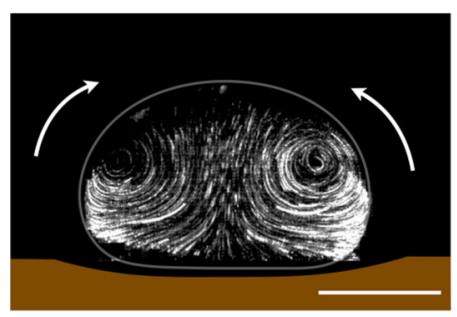

(b)

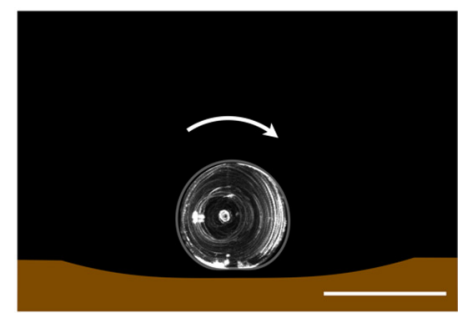

FIG. 1. Long-exposure photographs (exposure time is $200 \mathrm{~ms}$ ) of water drops levitating above slightly curved brass brought to $T=300^{\circ} \mathrm{C}$. Tracer particles have been added to visualize internal flows in the enlightened central planes. (a) A puddle hosts two internal convective cells. (b) Flow symmetry is markedly different for a quasispherical droplet, where we observe a simple rolling motion. Both bars show $2 \mathrm{~mm}$.

Published by the American Physical Society under the terms of the Creative Commons Attribution 4.0 International license. Further distribution of this work must maintain attribution to the author(s) and the published article's title, journal citation, and DOI. 
(a)
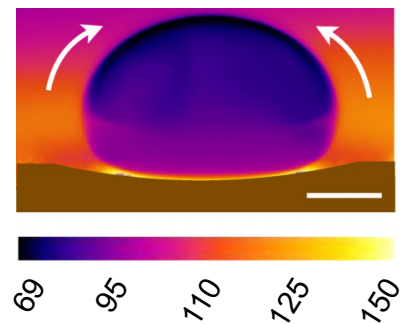

(b)

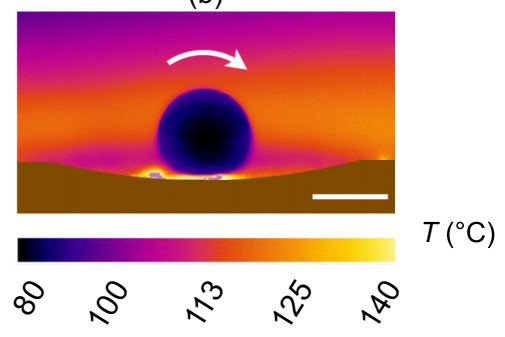

FIG. 2. Infrared images of the surface of a water drop (water is opaque at such wavelengths) levitating above a slightly curved brass surface heated at $350{ }^{\circ} \mathrm{C}$. Images are taken with a thermal camera using a calibration range from -40 to $150^{\circ} \mathrm{C}$, only suitable for water and not for brass. Both bars show $2 \mathrm{~mm}$.

The morphology of the inner flows depends on the drop size. On the one hand, water motion in a large drop consists of symmetric convective cells in the illuminated slice [Fig. 1(a)]. On the other hand, smaller droplets host a unique asymmetric flow cell [Fig. 1(b)]: water now simply rotates in a solidlike fashion.

The dynamical nature of Leidenfrost drops may originate from the vapor film whose flow can entrain liquid by viscosity, but it can also be temperature-driven [2]. Figure 2(a) reveals the existence of strong vertical thermal gradients along the drop surface, which induces interfacial Marangoni flow from the hot base to the cooler apex, with a downward motion along the drop vertical axis, a scenario in agreement with the flow characteristics reported in Fig. 1(a). In contrast, temperature is observed in Fig. 2(b) to be homogenized in smaller droplets, an effect that might be due both to the asymmetric rolling and to the proximity of the hot plate.

As is obvious in Figs. 1 and 2, large drops are flattened by gravity whereas smaller ones are quasispherical. This change in aspect ratio triggers the transition between symmetric counterrotation to asymmetric rolling-as also reported for other systems [3]. Interestingly, this symmetry breaking dictates the behavior of free drops on hot substrates made as horizontal as possible. Then symmetric puddles slide along the unavoidable slope of the substrate, while droplets are all observed to self-propel in a random direction that corresponds to their internal rotational (Fig. 3). This conversion of rotation into translation is not trivial, since drops rotate above a vapor film. We discuss in [4] the possible origin of the motion, which contributes to and even somehow explains the legendary mobility of liquids in the Leidenfrost state.
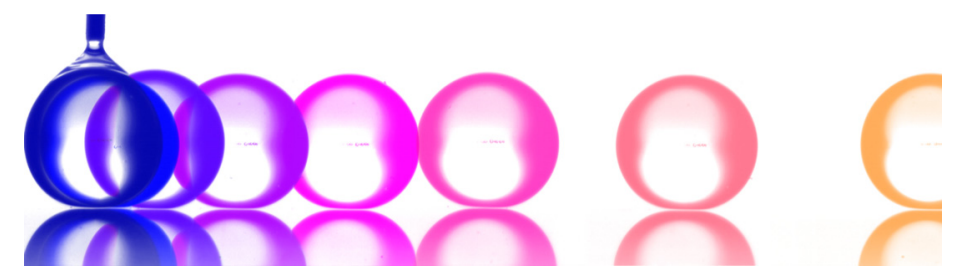

FIG. 3. Chronophotography of a millimeter-sized water droplet $(R \approx 1 \mathrm{~mm})$ self-propelling on a flat reflective substrate heated at $300{ }^{\circ} \mathrm{C}$. Water, initially immobilized by a needle located at a distance $H$ above the substrate, detaches at a radius $R \approx H / 2$ and then spontaneously moves away in the direction set by the internal rotation. The color gradient is used to highlight the successive positions of the drop (time interval of $\approx 180 \mathrm{~ms}$ ). 
[1] J. G. Leidenfrost, De Aquae Communis Nonnullis Qualitatibus Tractatus (Ovenius, Duisburg, 1756), Chap. XV; translation: C. Wares, On the fixation of water in diverse fire, Int. J. Heat Mass Transf. 9, 1153 (1966).

[2] D. Tam, V. von Arnim, G. H. McKinley, and A. E. Hosoi, Marangoni convection in droplets on superhydrophobic surfaces, J. Fluid Mech. 624, 101 (2009).

[3] S. Dash, A. Chandramohan, J. A. Weibel, and S. V. Garimella, Buoyancy-induced on-the-spot mixing in droplets evaporating on nonwetting surfaces, Phys. Rev. E 90, 062407 (2014).

[4] A. Bouillant, T. Mouterde, P. Bourrianne, A. Lagarde, C. Clanet, and D. Quéré, Leidenfrost wheels, Nat. Phys. (2018), doi:10.1038/s41567-018-0275-9. 